\title{
Forest genetics: pattern and complexity
}

\author{
Gene Namkoong
}

\begin{abstract}
The study of gene effects requires both reductionist and integrationist approaches to discern even simple effects as well as the multiple effects of single genes and the effects of multiple genes on phenotypes. Evolutionary genetics also requires use of both approaches to discern the mixed patterns of genotypic distributions that we observe in forest tree species. While this evolutionary dynamic does not necessarily generate a stable system, it has generally provided a diversity that can be used to design efficient breeding and conservation programs. The management of such programs, however, also requires us to develop a conservation ethic.
\end{abstract}

Résumé : L'étude des effets des gènes requiert à la fois des approches réductionniste et intégrative afin de discerner même les effets simples aussi bien que les effets multiples de gènes individuels et les effets de gènes multiples sur le phénotype. La génétique évolutive s'appuie également sur ces deux approches afin de discerner les patrons mixtes de distribution des génotypes observés chez les espèces d'arbre forestier. Même si cette dynamique évolutive n'engendre pas nécessairement un système stable, elle a généralement fourni une diversité pouvant être employée pour élaborer des programmes efficaces de croisement et de conservation. Toutefois, la gestion de ces programmes requiert également le développement d'une éthique de la conservation.

[Traduit par la Rédaction]

\section{Introduction}

In the study of complex systems, a common approach is to simplify parts of the system that we believe are separable to understand some of the segments and to reduce the system to its separate components. As a research method, this reductionism can be an insightful path to knowledge. However, it always raises the question of whether the way that the parts are put together may itself affect function and that the organism as a whole is formed by interactions among the parts that generate results that are not predictable from studying the parts. For systems as complex as forests, the interactions can be necessary for the system to function, and therefore, the system itself can be understood only if the integration is studied. There is a constant tension between reduction and integration that, if both approaches are used, will lead to a more complete forest science. A motivation for study is to use that tension to better appreciate forests. This essay is an attempt to consider the development of forest genetics and how we might use that creative tension to develop a science and aesthetics of forests. The steps we have taken to understand genes and gene actions are first reviewed. Then, concepts of cause and effect of genes on population dynamics and the effects of populations on genes are considered. Finally, the conservation and use of genetics in forestry is addressed in how biology and ethics can guide our actions.

A characteristic of forests is the complexity of effects that are involved in how they grow and develop. In fact, forests

Received November 22, 1999. Accepted November 11, 2000.

Published on the NRC Research Press Web site on

April 7, 2001.

G. Namkoong. Department of Forest Sciences, Forest Sciences Centre, 2424 Main Mall, University of British Columbia, Vancouver, BC V6T 1Z4, Canada

(e-mail: gene@interchange.ubc.ca). are the epitome of complexity. There are many different organisms that are competitors, mutualists, or users of trees, operating at many different time scales and generation intervals. They also affect and are affected by genetic processes, and this complexity is both the fascination of working with forests as well as the bane of trying to discern patterns that can direct us in conserving as well as using them. Especially in forestry, an impetus for research is to see order in complexity, to find rules of causation, and to understand how much of the complexity can be reduced to simple causes. There is an ontological reductionism in that populations are composed of their component individuals, which are in turn composed of their organs, tissues, cells, and genes. This is sometimes taken further to a methodological reductionism that implies that all function derives from the genes and that higher level of biotic organization are mere outgrowths that carry gene effects. Biological reductionism is then sometimes driven to considering forests only as a woodproduction system. In extreme reductionism, "selfish genes" are considered to control everything including individual function, and both forestry and genetics are equivalent to engineering.

In contrast, ecosystem and population dynamics operate at higher levels of biological organization and give order to complexity that cannot be discerned at reduced levels of organization. Meaningful function is defined by selection and evolutionary events at the individual and population level and genes accumulate because of their higher level effects. Research in genetics and forestry is therefore also directed to discerning the levels of aggregation and organization of lower level units at which interacting factors exert influences that are not present at lower levels of organization. Hence, we also seek to understand the features of higher biotic levels, which affect the functions of lower levels of organization. In the best kind of genetics we use both the reductionist and the integrationist approaches, either simultaneously or sequentially. For some geneticists, the approach may be first 
on individual gene processes, which can then be aggregated for multilocus effects in cells or tissues, and then further aggregated for the populations, species, and ecosystems in which they evolve. Alternatively, we may start at the level of the forest and the reproductive and competitive environment that defines fitness and determines how genes function in biotic communities. In fact, there are many entry points to our inquiries about forests and from any of them, we may take first a reductionist approach and then an integrationist, or vice versa. We may find that genes affect growth properties, but also that the properties of genes at one level are determined by their combined and integrated function in a developmental or ecological system. Gene effects may be emergent properties of molecular processes but they are also the result of functions that are partly determined by development imposed by developmental, population, and ecosystem processes.

However, understanding the complexity of gene actions and gene effects requires not only advanced chemistry, physiology, and dynamics but also requires that we consider many factors simultaneously. It requires incorporating the dimensions of multiple gene effects, the dimensions of gene and environmental factors, the dynamics of multiple populations and multiple species interactions, each with several factor dimensions. These multiple dimensions are necessary for understanding the complexity of gene effects and evolution as well as the existence of patterns in mechanics and evolution. However, the human capacity for managing multiple dimensions is limited. We rarely think beyond three or at most four dimensions, and hence, we are limited in our capacity to understand complex systems. We try to discern the rules and order within the complexity and the levels at which order can become apparent, but we also acknowledge that there are functions and biotic organizations that may forever remain hidden. We wish to avoid teleology and the imposition of causes that are only extensions of our ignorance, but we do not deny that ordering factors can exist whether we can know them or not. We have a philosophical problem in forestry and genetics in knowing if the existence of patterns in complexity is a reification of the human desire for order or if there is order and pattern that can be discerned because there is a design. A theological problem is whether there is a designer.

\section{Gene effects on individuals}

Starting at the level of the individual organism, gene effects have not been as clearly seen in forests as they have in other organisms. One of the obvious difficulties has been that large trees and long maturation times obscure simple gene effects and make field experimentation cumbersome and expensive. Performance could not be estimated on the kinds of difficult sites where soil heterogeneity was high and the costs of establishing tests limited the number of replications that could be afforded. As a result, tests were established on agricultural sites and were so large that high levels of site variability were included in a random or unexplained "error" even though environmental effects on development could, in principle, be separated from genotypic effects. Estimation was forced to be imprecise, and foresters could only know about gene effects observed as family or population (provenance) mean differences. We largely borrowed from Fisher's agricultural concepts of design and since then have been fortunate to have access to many books and programs on statistical design and estimation. While foresters have been slow to use efficient designs (Williams and Matheson 1994), the concepts of estimation efficiency have been made available. Optimum plot sizes (Conkle 1963) and quasi-balanced mating designs (Namkoong and Roberds $1974 a$ ) could be described for a range of heritabilities and for dual experimental objectives of estimating family means and for estimating variances (Pepper 1983). The use of unbalanced designs has also been introduced and alpha designs proposed to increase efficiency so that experiments could be efficiently conducted on variable field sites at large cost savings. The capacity of computers to find maximum likelihood estimators freed geneticists from the constraints and inefficiencies of balanced designs and now allows foresters to design more cost-effective experiments (McCutchan et al. 1989; Friedman and Namkoong 1986) and to explore how heterogeneity can be accommodated in design ( $\mathrm{Fu}$ et al. 1998).

With an increase in efficiency, more effects could be usefully estimated, and two research areas have since emerged: one statistical and one more genetic. The statistical problem is that we usually do not know what the actual parameters are going to be before we estimate them and, hence, must design for a range of parameter values. In addition, we usually wish to estimate the effects of several genetic and environmental components of variance and family means for several levels of environmental and genetic effects (Pepper 1983). We also usually have several traits that we wish to estimate simultaneously and, hence, need efficiency not only for different components but each over a range of parameter levels. If we wish to estimate how these factors interact, designs must be directed to a range of heritabilities and to estimate multivariate distributions. Several components for multiple traits are higher dimensions of design criteria, which are not theoretically new issues for statisticians but are not yet widely established in practice.

The second research area is more genetic in nature and follows from the observation that we can better understand and account for environmental heterogeneity in our experiments. It follows that the environment is less an unknown random variable, and the genetic variance will be a larger portion of the total variance. There should be an increase in heritability and a clearer understanding of the dependence between environmental and genetic effects. There are patterns to environmental effects that can be separated from random error, and if there is genetic variation in these patterns, they too can be described as gene effects. The environment then becomes a function of the genotypes that measure its effects, and we find that we cannot define an environment without the genotypes that measure it. In a deeper sense, we reaffirm Lewontin's (1974) observation that there also is no genotype without an environment and, vice versa, that the genotype defines the environment. In one attempt to define genotypic and environmental effects more rigorously, Gregorius and Namkoong (1987) introduced the concept of separability as necessary for an understanding of the gene- 
environment complex of the biological system. The patterns that emerge from the joint analysis reflect developmental and evolutionary constraints on the forms of response.

The agricultural model of a low-dimensional, univariate gene effect that can be estimated from a linear statistical model, however, remains as the paradigm for much of our present research and for the concepts of gene effects used in molecular genetics and QTL analyses. The analysis of gene actions, however, is also turning to the developmental feedback systems within which gene effects within the cell can be unstable with periodic or even chaotic behaviours. The definition and estimation of gene effects would have to be more than their functional parameters and their average effects and would have to include multivariate distribution parameters.

The concept of a gene or genotypic effect is thus dependent on the definition we use of the internal and external environments of inference and, hence, would vary depending on the level of biological organization at which we take our measurements. The environment, and hence the gene effect, depends on whether we measure at or below the level of the individual cell, or of the whole organism, or at the population and higher levels of observation. What can be defined as a gene effect then also depends upon the observer and what the observer considers to be significant. In this sense, we cannot understand the genotype without defining either the environment or the observer. I will return to this theme later, but one of the consequences of this for a general view of genetics is that, from the perspective of meaningful gene effects, there are multiple and interacting gene effects that produce any single phenotypic effect.

\section{Gene functions}

A further complexity of the concept of a gene effect is the developmental system, which involves interactions of several loci. It may in fact be questioned if there are any precisely definable single, major gene effects at all. Even at the subcellular level, genes that affect a trait are involved in interactions that determine the measured effect. Even when heritabilities and estimates of numbers of genes that affect a trait can be derived, epistasis is still found to be an important effect. Studies of some presumably simple, major gene effects in Drosophila, for example, indicate that, even when a system of major genes and modifiers can be estimated, development of chetae number is far from being a series of independent gene actions. Estimates of epistasis from linear gene effect models are obtained as departures from main effects and are low relative to their biological significance. Although difficult to estimate, epistasis is strong and complex (Mackay 1998). The general view for most traits must strongly favor the prevalence and strength of multiple-locus effects for any given trait. Research on this question could progress either from the perspective of using some simple systems to understand the variety of interactions of two, three, and more loci that occur and how they may be estimated. Alternatively, if interactions are the paradigm for gene actions, then general effects, which can still be defined for "genes," are not fixed or even estimable except as approximations or as average and variable effects.
We can continue to use the traditional estimates of gene effects with certain assumed conditions but can have confidence only for those constrained conditions. In general, gene effects at any useful level of organization would have to be indirect and involve epistasis of a high degree. Average effects can still be defined and used, but in an epistatic system, the effects would vary as other loci change in frequency. From generation to generation, the loci that strongly affect a trait, therefore, would vary, and the loci that are significant this generation shouldn't be expected to be the same loci or to have the same effects. With epistasis, fitness can be expected to have several peaks, and artificial selection can also maximize value with several different gene combinations. Optimality may require that multiple paths be explored and interpopulational variation patterns used, rather than seeking a single optimum. For both conservation and use, population sizes would have to be large enough to allow for the emergence of new gene combinations and effects.

If most heritable traits can be best understood as the combined effects of multiple loci, then each locus can potentially participate in reactions that affect several downstream developmental pathways. A general view of gene actions would have to include their effects on multiple traits. Unless a single locus has an effect only at the end of one developmental pathway, each locus must have pleiotropic effects involving several traits. Clearly any traits that operate in most physiological pathways would have multiple "effects" on any individual. Many of these effects may be considered to be trivial in terms of significance to our interests, but side effects must at least be considered for most genes. Therefore, genetic analysis must generally involve estimating multivariate responses, and pleiotropy must be generally assumed to exist. There is a distribution of gene effects that depend on the number and strength of gene effects on multiple traits. Research in molecular genetics is just beginning to go beyond single locus effects and is turning to understanding this functional level of gene action.

For this epistatic-pleiotropic concept of gene action, a completely reductionistic approach to understanding and managing gene effects is overly simplistic, and one of the great challenges for genetic research is finding the appropriate levels of definition and analysis. The problem is to find sets of genes that function in concert and that are separable such that set "effects" can be defined. How many such sets exist, and if there is a finite number of systems, at what level of physiological development can their functions be described? If single loci operate on more than one system, then how many systems can they have and still retain a clearly definable gene effect? There is more than one effect per gene, and gene function depends on the developmental systems that function at the individual and higher levels of biotic organization. The higher level functions may then be understood to affect genes and hence that gene effects exist, but they are not the causes of function. This is not meant to imply a belief in a totally "holistic" or deterministic and teleological view of genetics either, where everything is assumed to be tightly integrated for a specific purpose and no freedom exists for any of the forces of evolution. Rather, it is intended to focus research attention on the multiple levels and scales of organisms at which we can understand, define, 
measure, and manage the genes. Gene effects are measurable, but on how they are measured and in the future will require multidimensional definitions and analyses. Epistasis and pleiotropy are the rule, not the annoying complication, implying that breeding and evolution should assume that multiple peaks of adaptation and value generally exist.

We must now define a gene effect in terms of its degrees of independence and the dimensions of its influence on multiple traits as well as its effects on any single measure. Estimates of gene effects will not be limited to point estimates but will be distributional. By probing the molecular dynamics of genes, we may begin to derive such measures and observe patterns of gene effects at levels of collective gene action and among correlated, multiple effects. These measures of gene effects will require multiple dimensions to detect and ascribe patterns in both causes and effects. But with higher dimensions of cause and effect, we also have to develop our capacity to work with more than three or four dimensions simultaneously. In the study of multiple allelic effects in multiple environments that affect multiple traits, at this time we do not have either the concepts or the tools that allow us to understand higher dimensions of complexity even though we can see that patterns lie in those dimensions. We can see average effects of genes, we can see that traits are genetically correlated and that development is a coordinated affair, but we cannot yet discern the degrees of independence among genes and traits.

\section{Genetic dynamics}

If we consider that multiple traits are affected by multiple loci, and that gene effects themselves have complex interactions that we may begin exploring at molecular and field levels, we can also consider that genes affect and are affected by population and species level events. Evolution then also becomes a study in ecosystem and evolutionary complexity that obeys different dynamic forces. Since simple selection effects can either tend to diverge otherwise uniform, intermating populations or to homogenize alleles among nonintermating and diverged populations, the resulting distribution of alleles depends on the balance between selection and migration. Since selection can be expected to vary among loci because of both the gene effects themselves as well as to the environmental pattern of selective pressures, the balance of selection and migration must be expected to differ among loci in any set of populations (Mangold and Libby 1978). Some alleles at some loci will change in frequency because of a change in selection pressure along one or another environmental gradient. Some traits will respond to different selective environmental factors, while others will experience a broadly similar selection pressure even if reproductively isolated.

Still other traits will have little selection pressure at all, and their distribution would reflect only migration and population size effects. The conservation of such selectively neutral alleles only involves managing population sizes and is influenced by mutation and migration effects on the effective population size. Neutral models have served as the null cases for describing allele distributions and probabilities of extinction. For conservation purposes, mixed patterns must be considered to be the general rule, and what is conserved may be a mixture of distributions with a single criterion for population selection, or separate sets of populations, that optimize different diversities. It is also possible to use restrictions on migration to create more diversity than presently exists among populations, and to reveal patterns of divergent selection that are now obscured by migration.

The relative magnitude of effects and the congruence of the areas in which the selection and migration effects occur can give different distributions of allele frequencies for useful alleles (Eriksson et al. 1993; Chevillon et al. 1998). The research on "provenances" in forestry has developed some sketches of how heritable variations exist among populations and has shown that species differ in their responses to similar environmental gradients and that the patterns of difference or similarity differ within species according to the trait being studied (Morgenstern 1996; Rehfeldt 1989; Matyas 1996). The effects of "hitchhiking" among loci, when neutral alleles at one locus are affected by selection at a closely related linked locus, must also be considered as leading to nonoptimal allele distributions. Therefore, Every population must be considered as being the resultant of a mixture of evolutionary forces (e.g., see Martinson 1996). With multiple evolutionary forces operating, the joint distributions of multiple loci are a mixture of different balances between migration, mutation, drift, and selection. There is, then, no single tree of relationships among populations, but rather, there are several networks of relationship. The patterns that exist in these higher dimension structures may themselves be important for conservation. Sampling for conservation purposes should consider allele distributions that may be related to migration or to any of several selection gradients, and the extremes of geographic and ecological variables may be targeted for attention.

To discern relationships among populations and species, it is necessary to find the dimensionality of relationships that can exist and that can be expressed in some index of complexity. Different similarities and distances can simultaneously exist, and each can be a significant part of evolution. When differences or similarity indices are used with marker loci or morphological traits and trees of relationship are constructed, it is often assumed that a single branching tree exists. More data and multivariate analyses are used with the implicit assumption that they cumulatively contribute to the precision of estimating that tree. If loci are subject to different patterns of association, however, then more data may only mix more and different patterns of association. There may be a finite number of independent patterns such that we might say that one set of loci is subject only to one set of evolutionary forces such as migration and population size. In some cases, another pattern may exist because of a strong selective force balanced with migration and drift, but the distribution of alleles would not be the same as for neutral loci. In reality, we would have to assume that the selection is also multidimensional and that the balance between selection and migration in forests is of a higher dimension than generally modeled. This would require estimating not one evolutionary tree but estimating a multidimensional network.

To understand the existence of patterns in complexity of gene distributions, we would then want to know the dimensionality of the number of trees or networks that have evolved or that we can manage. The generations of prove- 
nance tests of forest trees carries a wealth of information not yet fully tapped for such analyses. In forests with multiple species interacting and with relatively weak environmental management, environmental complexity would force genetic complexity. A single hierarchy must be expected to be the exception and cannot serve as a generally useful paradigmatic structure for estimation. A multidimensional network would have to be the estimation objective, and the patterns that may exist among them may then reveal structures of evolution and associations among forces that we cannot now observe.

In the light of such structural complexity and the mixed forces of evolution that give rise to multiple structures, the management of genetic variation for either conservation or breeding purposes is unnecessarily constrained if limited to single populations or to single dimensions. We can manage multiple populations as well as single populations to effect our conservation or use goals. Yet, most conservation research focuses on island biogeography as if populations do not interact, and conservation biology is limited to concerns on reproduction in finite population sizes. Similarly, breeding theory focuses on single populations and usually on single breeding objectives. Neither considers the management of multiple populations nor the uses that can be made of migration management in conjunction with selection and population size controls. For multiple environments and multiple trait understanding and management, all of the forces of evolution can be used for the management of the total genetic resource.

For forest tree species in particular where modern interspecies hybridization is commonly found, the structure of diversity may require study of hybridization in generating multilocus gene effects (Koshy and Namkoong 1996), but interpopulation and interspecies dynamics provide a more comprehensive genetic management system. This implies that evolutionary relationships, even if predominantly hierarchic, may include connecting lines among otherwise separate branches to form complex networks of relationship. For breeding purposes, this also implies that managing multiple populations can result in a broader use of the total genome or of several genomes. Hence, for single- as well as for multiple-population management, breeding can be considered as a form of controlled evolution, and there is no qualitative difference between our breeding and conservation programs. Although complex, breeding and conservation can be unified in practice as well as in theory, by using multiple forces of evolution to guide future evolution. In the applied programs of conservation as in the Mata Atlantica, or the Amazon basin, the complexity is further compounded since they must deal with mixtures of species that each have different geographic distributions and regeneration requirements.

However, a problem persists even if we can usefully analyze this more complex genetic system in forests, since we must also consider that the genes and the forest environment are coevolving. In forestry, where most species are spread over large areas, take long time spans to mature, and can be managed with relatively crude technologies, the unmanaged ecosystem exerts strong pressure on genetic processes. On the other hand, the genetically influenced reproductive capacity of the component species influences what can be re- generated and what genotypes can respond. Unless they are brought under joint study, we still have a reductionist view of science and research that results in a static view of genetics.

Patterns in genetic complexity may lie at still higher levels of organization. Thus, while direct gene effects may start at the enzyme level, the effects which we find significant to measure may be at the level of an organ or tissue, at the level of individual, or its population growth and survival rates, etc. As long as populations differ in the frequency of alleles, and that difference is associated with events at the population level of observation and dynamics, a gene effect is definable no matter how indirect its action. Patterns in gene effects and allele frequencies among populations may be observed at the whole forest level. While we may have to increase the levels of complexity of gene effects to understand what drives the system, there may still emerge patterns that are due to an integration of different dynamic forces among levels of organization. Thus, provenances may well vary in different patterns of allelic association because of mixed evolutionary forces. Analyses of single hierarchic trees would blend patterns, and therefore, to see and understand associations, we may require high dimension analyses. With the complexity of higher dimensions, however, there is the danger that increasing complexity will only lead to sterile analysis and will make life less, not more, understandable. The creative tension in research lies in balancing the necessary complexity with the functional patterns that can only be dimly perceived.

\section{Population dynamics}

It seems necessary to take a more dynamic view of forests and to shift focus away from understanding only the genes and genotypes themselves as goals of genetics in general and forest genetics in particular and towards the functions of genes in the evolution of managed and unmanaged ecosystems. Especially when using populations in semicontrolled forest environments, complexities of the biotic and inanimate environments strongly affect gene functions. Conversely, genes affect the relationships between competitors, mutualists, successors, and species that otherwise coexist. Although gene effects are less direct than effects measured within individuals, effects of the ecosystem level may be more significant for evolution.

In the organization of forests, it is often assumed that there is a stable equilibrium inherent to a given landscape toward which the composition of a forest is being driven. Disturbances and human intervention are seen as deviant factors and stochastic variation is seen as an obscuring noise. One concept of biotic organization is that species-level events form the only organizing principle. From this view, each species is "doing its own thing" and the apparent existence of associated groups of species in communities or ecosystems are accidents of migration and survival. Species groups are merely emergent associations of historic and ecological processes, and the apparent communities are reifications. Much research is directed to finding the factors that force succession or allow some species to find appropriate sites and to compete with others. At least theoretically, these factors can then be used to estimate the transition probabilities 
from one set of genes, populations, and species to another and to derive an ultimate stable state. On the basis of an assumed stability, the competition models of Tilman (1988) and others emphasized the importance of limiting soil factors in subdivisions of environments and the ultimate succession of species to their final states. The forest succession dynamic models of Shugart (1984) and others identified a broader array of environmental factors that would eventually force a selective determinism to an evolutionary optimum. Our forest growth models use these concepts to predict the changes that can come as forests evolve and to determine what stable states may eventually equilibrate. However, models of processes that include variation in the transition probabilities indicate an expanding variance in outcomes and an instability in composition (Namkoong and Roberds 1974b).

There are two assumptions underlying this research agenda: first, that there is a progression to an equilibrium and, second, that the equilibrium is stable and globally attractive. There is a strong belief in the existence of an ideal state toward which change "progresses" (Brandon and Rausher 1996). There is also a hidden assumption that the human and forest ecosystems are independent and that human intervention thwarts those natural and inherently good tendencies that have allowed forests to evolve. These assumptions then lead to an ethic of conservation that must minimize human effects and idealizes "naturalism" and leads to the naturalist fallacy. It also follows that there is an inherent conflict between conservation and use and hence to management, which necessarily separates conservation areas from production forests. However, if human evolution has been affected by forests and vice versa, then the "material" and the human are not independent.

Furthermore, evolution to a stable and globally attractive equilibrium is not an evolutionary axiom. In the study of dynamical systems it is possible to derive conditions that would lead to inherent dynamics that do not necessarily evolve toward stable equilibria. With competition or frequencydependent selection among competing species or predatorprey relationships, the existence of genetic variation in responses can cause populations sizes and gene frequencies to form a joint evolutionary system. A wide variety of dynamic behaviours can exist for simple models of density-dependent competition that can be mediated by genes that affect competitive ability (Namkoong et al. 1993; Selgrade and Namkoong 1984). Genes that directly affect the acquisition and allocation of energy among plant parts can affect density and regeneration probabilities, which in turn can cause equilibria to destabilize and populations to develop periodic behaviour instead (Bishir and Namkoong 1991). Several kinds of attractors including strange attractors may exist in such systems (Sakai 1997; Selgrade and Namkoong 1992), and there can be an evolution of alleles toward the destabilization of population densities that is driven by genetic evolution (van Coller 1997). The relative densities of competitors in the ecological system are dependent on how the gene frequencies are evolving, and neither the competitive ecosystem nor the genetic system can be understood without the other. Genetic-ecological systems can evolve in which no equilibrium even theoretically exists. For a genetic management system, this implies that there may be no ideal, stable state that can serve as an objective for conservation, restoration, or for optimization.

In an interacting set of species, if one of them forces a periodic or chaotic pattern by its own density dynamics, then all members of the set would be forced to develop responses to an unstable, interactive biotic environment. The general behavioural rule for the whole system of populations and species would then not be driven toward a stable equilibrium. There is no "natural" tendency towards an ideal state in any of the participating species. A research problem is to detect when the factors that help determine system behaviour in fact lead to the various types of dynamics. At this time, it is not obvious when there actually may be stable points nor how we can distinguish unstable behaviour from stochastic variations in demographics or in the interaction parameters. The development of indices for detecting patterns that deny the existence of chaos would be important for conservation

The existence of chaos does not necessarily imply extinction, even if the dynamics approach chaos, since the dynamics may still be contained within self-generated limits. The dynamics of such systems and the protection of populations and species from extinction boundaries are also areas of research that are still to be developed. The conservation objective may be better directed to avoiding extinction rather than to seeking a fictive ideal and stable state. Populations and species may have inherent processes that may be chaotic, but which also avoid extinction, by compensatory behaviour at low population levels, which puts bounds on the dynamics near extinction. However, it is often difficult to detect whether variations in species composition, population size, or allele frequency occur because of deterministic or random variations. Therefore, even for systems that appear to be random in behaviour, deterministic forces may still exist that can force patterns in their variations to form. The discovery of pattern from the complexities of multiple-species interactions and its distinction from true randomness has been a persistent problem in ecology but not yet applied to genetic phenomena. The detection of nonrandomness in these dynamical systems is a biological-mathematical problem with large theoretical and practical significance.

If pattern exists at the forest level, then the origin of pattern may be an emergent effect of independent, lower level elements such that each population and species is "doing its own thing" and pattern is a crudescence without meaning. Alternatively, pattern may emerge, because there are functions of a larger biotic system that create strong interactions among the elements. In that case, species assemblages are not random associations without cause, but they are selected and have mutualistic interactions that support their existence. This may be more easily perceived at the level of the individual organism where major selection events occur or in populations where survival is dependent on mutual effects for development and reproduction. Genes in individuals are not random operators that happen to produce whole trees, but they are assembled because they function together. Populations may grow and survive not as random collections of individuals, but their collective capacities induce certain kinds of competition and cooperation. In spite of the strong tendency to oversimplify these arguments and for holistic arguments to claim absolute linkages, the scientific challenge 
is to find the levels and strengths of independent and integrative functions. For forest managers, it implies that management for whole forests is an appropriate objective and that it is not the same as managing for its individual elements.

In forestry, this is a particularly difficult task. To conserve forests as complex, interacting, ecological-genetic systems requires that cohort interactions be investigated and that multiple trophic levels be included. Hence, the bacteria, invertebrates, fungi, and higher life forms must be considered as part of the system and not as pests to be ignored or eliminated. The dynamics of the interactions with their different time and spatial scales is difficult and complex but can be approached (Levin 1978), and management can be directed to avoidance of extinction boundaries. The rhythms of forest systems may then be the objects that we wish to conserve and not primarily the individual elements. Biodiversity may then form a conservation objective that is above the level of individual species and its conservation would require the use of multiple sites, various management practices, and multiple time scales. Just as normal forest management objectives would be expected to shift as economics and the environment shifts, biodiversity management would also be expected to shift the location of reserves and their methods of management.

The practicalities of management may also require that to conserve species, a species-by-species approach cannot succeed and, therefore, requires that conservation planning at the "ecosystem" level is needed. If the biology of such systems is actually to be conserved, however, biologists must investigate how and if the multiple species system can in fact be kept from the edges of extinction. Since we can never have either very complete knowledge or very secure control, the use of multiple and replicate sites would be optimal for both security as well as for scientific understanding. Practicality as well as conserving the principal forces of evolution may force us to manage whole ecosystems with multiple compositions. This is not meant to imply that we must conserve everything in its present state or in what we might think are desired future conditions. It is meant to suggest that management targets sets of wide diversity such that an ensemble is accumulated that gives future management maximum capacity to adapt to whatever future conditions may require. This ensemble can be a multidimensional set, which can then be used as a foundation for managing a future with greater diversity than currently exists.

To further complicate our views of forests, an historic dimension must also be recognized as a nonrandom causative factor. Events such as migration and the order of arrival may be accidents of history that have little to do with deterministic factors but that can determine subsequent species associations and evolution of forests. Postglacial migrations from refugia around barriers are known to have influenced species compositions and stochastic displacement events imply that there are equally feasible past histories for a given area. There is a distribution of feasible histories, and we cannot know which among several possible outcomes may even have been more likely to occur. Just as there can be alternate futures that we can influence, there may have been alternate histories that could have influenced our present choices. We do not have the luxury of experiencing replicate lives, but it would be useful to consider the existence of alternate histories and whether the one we think we have experienced is random or optimal in some sense. Would we have predicted the paths taken, can we then predict the path we will take, and what do we perceive as the forcing factors?

Another limit to our ability to finely manage forests is that human influence in forests and forest influences on human development are so strong that we cannot independently experiment on forests without affecting human interactions. We have no independent controls for experimentation, and hence, our observations are always conditional on historic human-forest interactions. Recent research indicates the pervasiveness of purposeful human effects on forests in the socalled primitive societies and, hence, that the interactions of humans and forest vegetation imply an evolutionary interdependence. The complexities of human-forest interactions increases the dimensions of variability that can exist and is part of the forest system that management must consider. The patterns or design of complexity between the "natural" and human world requires a joint management in principle and not just for practicality. The design of conservation programs would have to include variation in how humans have interacted in forests. An emerging view of the human-forest interaction is that there are no forests without a long history of human influence and that, by withdrawing human effects, we are changing human effects and not restoring a reified pristine state. The question for us is how to manage and is not whether we ought to manage. We never had the option of not managing, and any choice to withdraw management is a choice that has consequences that are as predictable or unpredictable as any other management choice. We may wish to choose minimum interventionist techniques for at least some management areas, but this should be part of a designed set of treatments.

In spite of the difficulties in understanding the extent to which driving forces versus historic and stochastic variations affect the emergence of patterns of association at the genetic and ecosystem levels, we are forced to manage finite sets of ecosystems. Given the uncertainties of our capacity to understand high-level dynamics and historical contingencies, the use of multiple populations increases the possibilities for reaching useful evolutionary states.

A research agenda for forestry may however be strictly reductionistic and would be directed to finding the one optimum condition. This is based on the concept that pattern is only an emergent property of lower level processes and that our observations of pattern are not assignable to higher level causes or are reifications of human wishes for order. Alternatively, pattern may be due to forces that require certain associations to form and not others. In our ecological-genetic system, this would most certainly require that interactions at several levels force genes, individuals, species, and ecosystem, to be ordered in multidimensional associations. If pattern exists for reasons other than random crudescence, then there is a design, and there may even be a designer, which is a theological question where science and religion can intersect.

Research could also be expanded into the aesthetics of forests that goes beyond visual attractiveness. If aesthetics is taken to mean the appreciation of mental images and the concepts evoked by a subject, then research into complexity 
can increase our appreciation of forests. There is a beauty in patterned complexity that can be seen in forests that underlies the visual appreciation of the physical forest that we can observe.

In the presence of multiple levels of interactions and multiple dimensions of causes and effects, an agenda for forest science as well as for conservation and optimum use is to explore those higher dimensions of existence and to enrich what we can know of forests and of ourselves. The scientific agenda can be built around the complexity and how to manage complex and irreducible systems to enlighten us about the levels of organization and order as juxtaposed to complexity and chaos. A temptation in science is to exclusively follow the reductionist strategy and to consider single dimensions of gene effects and ecological factors as if single cause-effect relationships are all there is to the ecologicalgenetic system. From this perspective, it also follows that we can simplify the forest to engineered products and processes. Even if that were possible, is it desirable? Can the science of the ecological-genetic system help inform what ethical management goals can be defined?

\section{Ethics}

Since human actions have been intimately involved with forests over the past one or two million years, and no forest can be considered to be separate from human activities, we must now more consciously manage that coevolved system. In the past, we may have had the luxury of ignorance of our effects, but we must now recognize that we influence evolution and can choose what those effects can be. We cannot withdraw management and assume that, in principle, we are not influencing a particular direction for forest evolution that is any less interventional than any other forest treatment.

Even if we consider human welfare, the ethics of conservation is not simple. Ethical issues involve the justice and fairness of choices that affect people in different social, economic, or political conditions that would then affect the evaluation of management decisions. Issues of local versus distant costs and benefits, different social condition, and how future generations can be considered are very complex in themselves. Similarly, the ethics of breeding or gene management involves questions of how different individuals and different segments of society can benefit from management. Present efforts at forest conservation often reflect values of dominant economic powers or the counter-culture, preservationist counter forces, neither of which brings any higher level of justice to the people affected by management. In the present climate of economic globalization, issues of justice have less effect on management, and there is a tendency to develop a segregation of interests into sociology and human ethics on the one hand in which biology has little input. The interaction between biology and ethics, however, can be enriched by reasoned analysis. At least two approaches can be taken; the first is to extend concepts of rights to organisms that possess morally relevant properties such as sentience. A second is to consider that even so abstract an entity as an ecosystem may have intrinsic values, even if we can only dimly perceive what they might be.

One of the first problems is to consider the subjects for whom we manage. The ethical obligations we have depend on the organisms for whose good we manage and which good we manage and which others can be managed as instruments for attaining that good. The question is to define what is included in the community of organisms where intrinsic values exist, what those values are, and what can we manage to protect that good. An issue in conservation ethics has been whether individual nonhuman organisms, or genes, or ecosystems can have anything but instrumental value to humans who have intrinsic values that we are ethically obliged to protect.

How can the scope of ethical inquiry include other than what we can know of obligations to humans? Can there be an ethics of the environment in contrast to an ethic about the environment? That is, do objects other than humans have rights and how can nonhuman beings have intrinsic values that must be respected? By arguing on the basis of rights, the extension of obligations to organisms that belong to other species often comes to a question of relative interests that contending individuals have in a management decision. Since most management decisions that may need an ethical perspective will involve conflicts of interests, one kind of rule that T. Regan develops is that the course of action be chosen that overrides the least amount of vital interests. In this context, biological research is necessary to inform ethicists about feasible options and reasonable consequences of management and the properties of the organisms involved (Namkoong and Regan 1988). This affects our judgments of what significant "interests" may exist among different species and how conflicting interests can be ethically managed. These judgments can be based on the properties that we can observe or infer are possessed by the involved organisms. This is a biologically informable issue that requires a compilation of what properties exist for different organisms and how management decisions could affect them, then some optimizing form of management could presumably be chosen. This is a significant issue for conservation that ethical research will need to further develop.

Apart from judging what obligations may be adjudicated among individuals, the ethics of other levels of biotic organization from the gene to the ecosystem also require development. The biological or ethical question then also involves whether there is an ethic that differs among biotic levels of organization. Useful inquiry might focus on species classes with different morally relevant properties, on the existence of interests in them, and on the balance of interests between a group and individuals. This line of inquiry would address the issue of giving species moral standing without overriding individual considerations. We may require multiple moral dimensions for guiding management decisions.

Ethicists have also been largely unsuccessful in addressing the problem of whether ecosystems can have intrinsic values. The problems are obvious, especially since we have already concluded that there are no "natural" goals for conservation and especially that the present state of the ecologicalgenetic forest system is probably neither stable nor optimal in any sense. There are no externally definable conservation goals, such as suggested by Leopold's (1970) dictum that states that "A thing is right when it tends to preserve the integrity, stability, and beauty of the biotic community. It is wrong when it tends otherwise." This assumes the prior existence of a stable, diverse, and optimal ecosystem that we 
should emulate. If the assumption is false, then there is no particular set of fixed objectives and, hence, no externally defined set of management goals or value functions that can ever, in principle, be defined. This leaves the problem of defining management goals in the position of moral relativism dependent only on ephemeral human desires and political power and, hence, denies the possibility of a conservation ethic.

Nevertheless, we are forced to manage an unstable ecological-genetic system and must determine what feasible objectives we can and ought to pursue even without explicit or "natural" rules to guide us. Since it is difficult to understand how we can have obligations to genes, species, or groups that can override obligations to individuals, it is even more difficult to conceive of obligations to whole forests for the sake of the forest independent of human interests. However, one way that this might exist is that we must first establish that an interactive, coherent system in fact exists whether we know in detail its composition or dynamics or not. A minimal obligation is then due to an entity that we ought not harm if it is benign to other organisms and may hold value.

The existence of order out of complexity and our ability to grope towards the discovery of higher orders of integration implies that there are patterns that we have not yet discovered. Therefore, the complexity itself is indicative of higher orders of obligation that may exist when order, pattern, or function can be discerned. If there is design to the complexity that we can perceive no matter how dimly, we have an obligation to not abuse the complex system if avoidable. Management for these purposes would not simplify forests to manufacturing factories, nor for restoring or preserving a world that never existed. It would manage for a partially known system of complex interactions in which pattern and higher order design exist but are only partially known and controllable. It would recognize that multiple histories and futures may exist and that we should diversify multiple units for conservation and study.

The study of forest genetics has been a struggle between understanding complexity and seeing the patterns that give it meaning from the gene to the ecosystem levels of biotic organization. The emergence of patterns is taken to imply the existence of functions at high levels that we often can only dimly perceive. The tension that exists can be used to motivate our research and to stimulate thought on why forestry is a creative profession.

\section{References}

Bishir, J., and Namkoong, G. 1991. Density-dependent dynamics in size structured plant populations. J. Theor. Biol. 154: 163188.

Brandon, R.N., and Rausher, M.D. 1996 Testing adaptationism: a comment on Orzack and Sober. Am. Nat. 148(1): 189-201.

Chevillon, C., Rivet, Y., Raymond, M., Rousset, F., Smouse, P.E., and Pasteur, N. 1998. Migration/selection balance and ecotypic differentiation in the mosquito Culex pipiens. Mol. Ecol. 7(2): 197-208.

Conkle, M.T. 1963. The determination of experimental plot size and shape in loblolly and slash pines. School of Forestry, North Carolina State University, Raleigh. Tech. Rep. 17.
Eriksson, G., Namkoong, G., and Roberds, J.H 1993. Dynamic gene conservation for uncertain futures. For. Ecol. Manage. 62(1-4): 15-37.

Friedman, S., and Namkoong, G. 1986. Estimating family means using unbalanced designs. In Proceedings, 1986 IUFRO Joint Meeting of Working Parties on Breeding Theory, Progeny Testing, and Seed Orchards, 12-18 October 1986, Williamsburg, Va. North Carolina State University-Industry Cooperative Tree Improvement Program, Raleigh. pp. 457-463.

Fu, Y.B., Clarke, G.P.Y., Namkoong, G., and Yanchuk, A.D. 1998. Incomplete block designs for genetic testing: statistical efficiencies of estimating family means. Can. J. For. Res. 28: 977-986.

Gregorius, H.-R., and Namkoong, G. 1987. Revolving dilemmas of interaction, separability, and additivity. Math. Biosci. 85: 51-69.

Koshy, M.P., and Namkoong, G. 1996. Futuristic breeding-some plausible options. In Tree Improvement for Sustainable Forestry. Proceedings, QFRI-IUFRO Conference, 27 October - 1 November 1996, Caloundra, Queensland, Australia. Edited by M.J. Dieters, A.C. Matheson, D.G. Nikles, C. Harwood, and S.M. Walker. Queensland Forest Research Institute, Caloundra, Australia. pp. 333-337.

Leopold, A. 1970. A Sand County almanac, with essays on conservation from Round River Ballantine, New York. pp. 202-210.

Levin, S. 1978. On the evolution of ecological parameters. Ecological genetics: the interface. Springer-Verlag, Berlin.

Lewontin, R.C. 1974. The genetic basis of evolutionary change. Columbia University Press, New York.

Mackay, T.F.C. 1998. The nature of quantitative variation: lessons from Drosophila. In Proceedings of the 6th World Congress on Genetics Applied to Livestock Production, 11-16 January 1998, Armidale, Australia. Vol. 26. Animal Genetics and Breeding Unit, Armidale, Australia. pp. 69-76.

Mangold, R.D., and Libby, W.J. 1978. A model for reforestation with optimal and suboptimal tree populations. Silvae Genet. 27(2): 66-68.

Martinson, S.R. 1996. Association among geographic, isozyme, and growth variables for sugar pine (Pinus lambertiana Dougl.) in southwest Oregon and through out the species range. Ph.D. dissertation. North Carolina State University, Raleigh.

Matyas, C. 1996. Climatic adaptation of trees: rediscovering provenance tests. Euphytica, 92: 45-54.

McCutchan, B.G., Namkoong, G., and Giesbrecht, F. 1989. Design efficiencies with planned and unplanned unbalance for estimating heritability in forestry. For. Sci. 35: 801-815.

Morgenstern, E.K. 1996. Geographic variation in forest trees. University of British Columbia Press, Vancouver.

Namkoong, G., and Regan, T. 1988. The question is not, "Can they talk?" J. Med. Philos. 13: 213-221.

Namkoong, G., and Roberds, J.H. 1974a. Choosing mating designs to efficiently estimate genetic variance components for trees. I. Sampling errors of standard analysis of variance estimators. Silvae Genet. 23: 45-53.

Namkoong, G., and Roberds, J.H. 1974b. Extinction probabilities and the changing age structure of redwood forests. Am. Nat. 108: $355-368$.

Namkoong, G., Bishir, J., and Roberds, J.H. 1993. Evolutionary effects of density-dependent selection in plants. Genet. Res. 62: $57-62$.

Pepper, W.D. 1983. Choosing plant-mating design allocations to estimate genetic variance components in the absence of prior knowledge of the relative magnitudes. Biometrics, 39: 511-521.

Rehfeldt, G.E. 1989. Ecological adaptations in Douglas-fir (Pseudotsuga menziesii var.glauca): a synthesis. For. Ecol. Manage. 28(3-4): 203-215. 
Sakai, S. 1997. Pioneer-climax tree competition models. M.Sc. dissertation, Institute of Applied Mathematics, University of British Columbia, Vancouver.

Selgrade, J.F., and Namkoong, G. 1984. Dynamical behavior of differential equation models of frequency and density dependent populations. J. Math. Biol. 19: 133-146.

Selgrade, J.F., and Namkoong, G. 1992. Dynamical behavior for population genetics models of differential and difference equations with nonmonotone fitnesses. J. Math. Biol. 30: 815-826.

Shugart, H.H. 1984. A theory of forest dynamics. The ecological implication of forest succession models. Springer-Verlag, New York.

Tilman, D. 1988. Plant strategies and structure and dynamics of plant communities. Princeton University Press, Princeton, N.J.

van Coller, L., and Namkoong, G. 1997. Periodic polymorphisms with density dependent selection. Gene Res. 70: 45-51.

Williams, E.R., and Matheson, A.C. 1994. Experimental design and analysis for use in tree improvement. Commonwealth Scientific and Industrial Research Organization, Victoria, Australia. 